\title{
Discovery of a radio relic in the low mass, merging galaxy cluster PLCK G200.9-28.2
}

\author{
Ruta Kale, ${ }^{1 \star}$ Daniel R. Wik, ${ }^{2,3,4}$ Simona Giacintucci, ${ }^{5}$ Tiziana Venturi, ${ }^{6}$ \\ Gianfranco Brunetti, ${ }^{6}$ Rossella Cassano, ${ }^{6}$ Daniele Dallacasa ${ }^{6,7}$ and \\ Francesco de Gasperin ${ }^{8}$ \\ ${ }^{1}$ National Centre for Radio Astrophysics, Tata Institute of Fundamental Research, Post Bag 3, Pune 411007, India \\ ${ }^{2}$ NASA Goddard Space Flight Centre, Code 662, Greenbelt, MD 20771, USA \\ ${ }^{3}$ The Johns Hopkins University, Homewood Campus, Baltimore, MD 21218, USA \\ ${ }^{4}$ Department of Physics and Astronomy, University of Utah, 115 South 1400 East, Salt Lake City, UT 84112, USA \\ ${ }^{5}$ Naval Research Laboratory, 4555 Overlook Avenue SW, Code 7213, Washington, DC 20375, USA \\ ${ }^{6}$ INAF-Istituto di Radioastronomia, via Gobetti 101, 40129 Bologna, Italy \\ ${ }^{7}$ Dipartimento di Fisica e Astronomia, Universita di Bologna, via Ranzani 1, 40127 Bologna, Italy \\ ${ }^{8}$ Leiden Observatory, Leiden University, P.O.Box 9513, NL-2300 RA Leiden, The Netherlands
}

Accepted XXX. Received YYY; in original form ZZZ

\begin{abstract}
Radio relics at the peripheries of galaxy clusters are tracers of the elusive cluster merger shocks. We report the discovery of a single radio relic in the galaxy cluster PLCK G200.9-28.2 $\left(z=0.22, M_{500}=2.7 \pm 0.2 \times 10^{14} M_{\odot}\right)$ using the Giant Metrewave Radio Telescope at 235 and $610 \mathrm{MHz}$ and the Karl G. Jansky Very Large Array at 1500 MHz. The relic has a size of $\sim 1 \times 0.28 \mathrm{Mpc}$, an arc-like morphology and is located at $0.9 \mathrm{Mpc}$ from the X-ray brightness peak in the cluster. The integrated spectral index of the relic is $1.21 \pm 0.15$. The spectral index map between 235 and $610 \mathrm{MHz}$ shows steepening from the outer to the inner edge of the relic in line with the expectation from a cluster merger shock. Under the assumption of diffusive shock acceleration, the radio spectral index implies a Mach number of $3.3 \pm 1.8$ for the shock. The analysis of archival XMM-Newton data shows that PLCK G200.9-28.2 consists of a northern brighter sub-cluster, and a southern sub-cluster in a state of merger. This cluster has the lowest mass among the clusters hosting single radio relics. The position of the Planck Sunyaev Ze'ldovich effect in this cluster is offset by $700 \mathrm{kpc}$ from the X-ray peak in the direction of the radio relic, suggests a physical origin for the offset. Such large offsets in low mass clusters can be a useful tool to select disturbed clusters and to study the state of merger.
\end{abstract}

Key words: shock waves - galaxies: clusters: individual: PLCKG200.9-28.2- galaxies: clusters: intra-cluster medium - radiation mechanisms: non-thermal - radio continuum: general - X-rays:galaxies:clusters

\section{INTRODUCTION}

Radio relics are diffuse, elongated, radio sources of synchrotron origin, occurring at the peripheries of galaxy clusters in the form of spectacular single or double symmetric arcs around cluster centres (e.g. Bagchi et al. 2006; Giacintucci et al. 2008; van Weeren et al. 2010; de Gasperin et al. 2014). These are exclusively found in clusters undergoing mergers and have been proposed to originate in acceleration/ re-acceleration at merger-

^ E-mail:ruta@ncra.tifr.res.in shocks (see Brunetti \& Jones 2014, for a review). The scenario is supported by several observational facts: The radio spectral index distribution across relics typically shows a flat to steep trend from the outer to the inner edge (e.g. Orrú et al. 2007; van Weeren et al. 2010, 2012; Kale et al. 2012; Bonafede et al. 2012; Stroe et al. 2013; de Gasperin et al. 2015). Relics have been found to be polarized (20 - 30 per cent), indicating underlying ordered magnetic fields (e.g. van Weeren et al. 2010; Kale et al. 2012; de Gasperin et al. 2015).

Diffusive shock acceleration (DSA) of electrons at shocks driven by merging sub-clusters can explain the 
power-law spectra of radio relics (e. g. Enßlin et al. 1998; Hoeft \& Brüggen 2007; Kang \& Jones 2005; Kang et al. 2012). However, DSA has low acceleration efficiency in the low Mach number shocks $(M<4)$ typical in galaxy clusters (e. g. Markevitch 2010) and thus, is not sufficient to explain some of the observed large radio relics (Brunetti \& Jones 2014). The observed spectral curvatures and widths of a few radio relics cannot be explained under standard DSA (e. g. Stroe et al. 2013; Ogrean et al. 2014; Trasatti et al. 2015; Stroe et al. 2016). The contamination due to the SunyaevZe'ldovich (SZ) effect can explain the spectral steepening in some relics (Basu et al. 2016).

Recently, using the particle-in-cell simulations of weak collisionless shocks, Shock Drift Acceleration (SDA) has been proposed as a pre-heating mechanism (e. g. Matsukiyo et al. 2011; Guo et al. 2014). This can alleviate the issue of low acceleration efficiency with the standard DSA providing a first stage of re-acceleration. The remnants of radio galaxies available at the location of the shock are also plausible sources of seed relativistic electrons to DSA (e. g. Markevitch et al. 2005; Kang \& Ryu 2015). However the role of magnetic field, the efficiency of acceleration, and injection of seed relativistic electrons in the shock is still not well understood (Brunetti \& Jones 2014).

Radio relics are known to occur as single or double arcs at cluster peripheries. Double relics are special systems where the associated cluster merger axis is nearly in the plane of the sky and allows the detection of the shock properties in radio and X-rays. For the sample of known double radio relics a scaling relation between the $1.4 \mathrm{GHz}$ radio power of the relic and the host cluster mass has been reported by de Gasperin et al. (2014). It was also shown that the single relics show a large scatter around this scaling relation. Galaxy clusters with single arc-like relics may be cluster mergers with a geometry unfavourable to allow the view of the second relic or are intrinsically systems with merger parameters that did not result in the generation of the second relic. A large sample of relics is needed to understand the connection between mergers and generation of relics.

The number of known relics associated with merger shocks is still small. Searches for relics have been carried out in all sky radio surveys such as the NRAO VLA Sky Survey (NVSS Condon et al. 1998; Giovannini \& Feretti 2000), the Westerbork Northern Sky Survey (WENSS Rengelink et al. 1997; Kempner \& Sarazin 2001) and the VLA Low Frequency Sky Survey (VLSS Cohen et al. 2007; van Weeren et al. 2009) in the directions of galaxy clusters. Radio relics have also been discovered using observations with the Green Bank Telescope in the directions of clusters (Farnsworth et al. 2013). In the Extended GMRT Radio Halo Survey sample that consisted of clusters with X-ray luminosity $>5 \times 10^{44} \mathrm{erg} \mathrm{s}^{-1}$ in the redshift range of $0.2-0.4$, the radio relics were found in $\sim 5$ per cent of the clusters (Kale et al. 2015).

Galaxy clusters with indications of an ongoing merger are potential candidates as hosts of radio relics. Recently the Planck satellite, using the SZ decrement, has detected 947 clusters of which 214 clusters are new (Planck Collaboration et al. 2015). The newly discovered clusters have lower luminosities and flatter density profiles as compared to the clusters already known from X-ray observations (Planck Collaboration et al. 2012, hereafter PC12).
Table 1. Properties of PLCK G200.9-28.2 (PC12).

\begin{tabular}{ll}
\hline RA $_{\mathrm{J} 2000}$ & $04 \mathrm{~h} 50 \mathrm{~m} 20.9 \mathrm{~s}$ \\
DEC $_{\text {J2000 }}$ & $-02^{\circ} 56^{\prime} 57.6^{\prime \prime}$ \\
Redshift & 0.22 \\
$L_{500,[0.1-2.4] \mathrm{keV}}$ & $(0.99 \pm 0.04) \times 10^{44} \mathrm{erg} \mathrm{s}^{-1}$ \\
$M_{500}$ & $(2.7 \pm 0.2) \times 10^{14} \mathrm{M}_{\odot}$ \\
\hline
\end{tabular}

These properties indicate ongoing mergers and therefore the new Planck clusters are potential candidates for the search of radio relics in radio surveys.

Radio relics have been reported in the new Planck clusters since the Planck Early SZ catalogue of galaxy clusters (Bagchi et al. 2011; de Gasperin et al. $2014,2015)$. In this paper, we report the discovery of a radio relic at the periphery of a new Planck cluster, PLCKG200.928.2, using radio observations from the Giant Metrewave Radio Telescope (GMRT) and the Karl G. Jansky Very Large array (VLA). The paper is organized as follows: The galaxy cluster PLCK G200.9-28.2 is introduced in Sec. 2. The radio data analysis and the images are presented in Secs. 3 and 4. The analysis of XMM-Newton data towards this cluster is presented in Sec. 5. The origin of the radio relic is discussed in Sec. 6. Conclusions are presented in Sec. 7 .

We adopt $\Lambda$ CDM cosmology with $H_{0}=71 \mathrm{~km} \mathrm{~s}^{-1} \mathrm{Mpc}^{-1}$, $\Omega_{\Lambda}=0.73$ and $\Omega_{m}=0.27$ in this work. This implies a linear scale of $3.52 \mathrm{kpc}$ per arcsec at the redshift of the cluster PLCKG200.9-28.2.

\section{PLCK G200.9-28.2}

PLCK G200.9-28.2 was a candidate cluster in the Planck satellite Early Cluster catalogue (Planck Collaboration et al. 2011a, hereafter PC11) that was subsequently confirmed with the $X M M$ Newton observations in X-rays (PC12). It is a cluster at a redshift of 0.22 with mass $M_{500}=(2.7 \pm 0.2) \times 10^{14} M_{\odot}$, X-ray luminosity $L_{500,[0.1-2.4] \mathrm{keV}}=(0.99 \pm 0.04) \times 10^{44} \mathrm{erg}$ $\mathrm{s}^{-1}$, and an average temperature of $4.5 \mathrm{keV}$ (Table 1). This cluster has a large offset of $3.4^{\prime}$ between the X-ray and the SZ position, which is above the median offset of 1.6' (Planck Collaboration et al. 2011a,b) for other clusters in the Planck catalogue and the Planck reconstruction uncertainty that peaks at $2^{\prime}$ (PC12). An extended radio source at the edge of this cluster was noticed in the NVSS and was identified as a candidate radio relic (Kale et al. 2013). In this work, we present deep radio observations and a re-analysis of the X-ray data that allow us to study the nature of the radio relic and the host cluster.

\section{RADIO DATA ANALYSIS}

We have observed PLCK G200.9-28.2 with the GMRT and the VLA in Dec. 2012 and Jul. 2013, respectively. The summary of the observations is given in Table 2 . 
Table 2. Summary of the radio observations.

\begin{tabular}{cccccccc}
\hline Telescope & Date & $\begin{array}{c}\text { Freq. } \\
\mathrm{MHz}\end{array}$ & $\begin{array}{c}\text { BW } \\
\mathrm{MHz}\end{array}$ & $\begin{array}{c}\text { Freq. res. } \\
\mathrm{kHz} / \text { channel }\end{array}$ & $\begin{array}{c}\text { Time } \\
\text { min. }\end{array}$ & $\begin{array}{c}\text { Beam } \\
\text { " }{ }^{\prime \prime},{ }^{\circ}\end{array}$ & $\begin{array}{c}\sigma_{\mathrm{rms}} \\
\mathrm{mJy} \mathrm{beam}^{-1}\end{array}$ \\
\hline GMRT & 31 Dec. 2012 & 235 & 8 & 130 & 390 & $14.7 \times 12.8,50.4$ & 0.39 \\
GMRT & 31 Dec. 2012 & 610 & 32 & 130 & 390 & $5.6 \times 4.7,59.4$ & 0.045 \\
VLA & 04 Jul. 2013 & 1500 & 1000 & 25 & 90 & $14.5 \times 10.0,33.7$ & 0.060 \\
\hline \hline
\end{tabular}

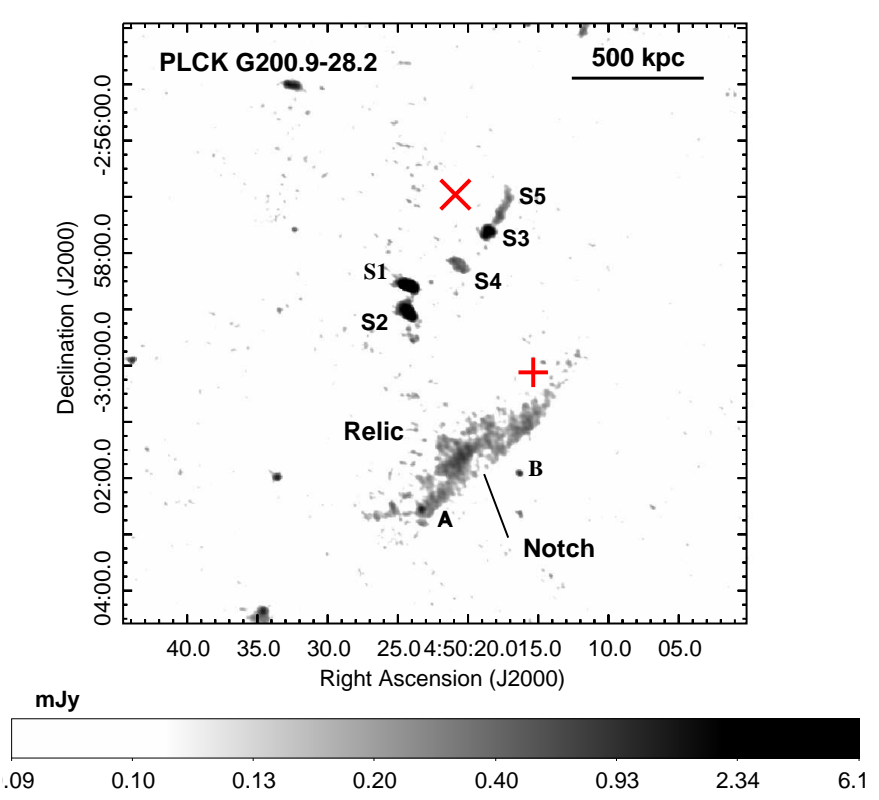

(a) GMRT $610 \mathrm{MHz}$

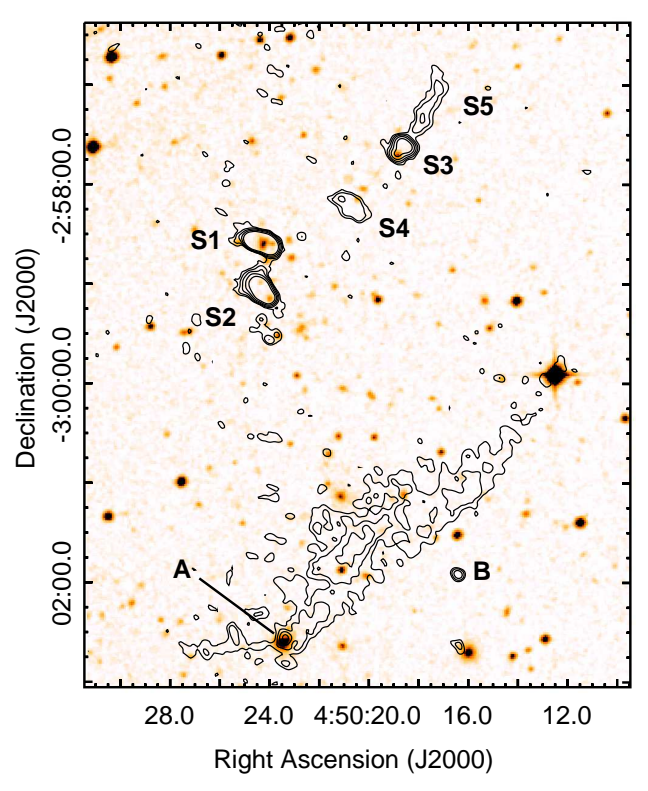

(b) GMRT $610 \mathrm{MHz}$ contours on DSS R-band

Figure 1. (a) - GMRT $610 \mathrm{MHz}$ image is shown in grey scale. The $610 \mathrm{MHz}$ image has a resolution of 5.6" $\times 4.7^{\prime \prime}$, position angle 59.4 and rms noise, $\sigma_{\text {rms }}=0.045 \mathrm{mJy}$ beam $^{-1}$. The discrete sources in the central region are labelled S1 $-\mathrm{S} 5$ and those near the extended source labelled 'Relic' are A and B. The symbols ' $x$ ' and ' + ' mark the positions of the cluster according to the XMM-Newton and the Planck measurements, respectively. (b) - Digitized Sky Survey R-band image of the region of the labelled sources is shown in grey scale with the GMRT $610 \mathrm{MHz}$ contours overlaid. The contour levels are $3 \sigma_{\mathrm{rms}} \times[ \pm 1,2,4, \ldots]$ mJy beam ${ }^{-1}$.

Table 3. Radio properties of the discrete radio sources in the PLCK G200.9-28.2 field.

\begin{tabular}{ccccccc}
\hline Source & $\begin{array}{c}R A_{J 2000} \\
\text { hh mm ss }\end{array}$ & $\begin{array}{c}D E C_{J 2000} \\
0 \prime \prime \prime\end{array}$ & $\begin{array}{c}S_{235 \mathrm{MHz}} \\
\mathrm{mJy}\end{array}$ & $\begin{array}{c}S_{610 \mathrm{MHz}} \\
\mathrm{mJy}\end{array}$ & $\begin{array}{c}S_{1500 \mathrm{MHz}} \\
\mathrm{mJy}\end{array}$ & $\begin{array}{c}\text { Spectral index } \\
\alpha\end{array}$ \\
\hline S1 & 045024.57 & -025834.6 & $149 \pm 15$ & $89 \pm 5$ & $49.4 \pm 4.9$ & $0.63 \pm 0.04$ \\
S2 & 045024.10 & -025907.6 & $70 \pm 7$ & $39 \pm 2$ & $18.5 \pm 1.9$ & $0.79 \pm 0.06$ \\
S3 & 045018.56 & -025736.6 & $25 \pm 3$ & $14 \pm 1$ & $8.4 \pm 1.0$ & $0.58 \pm 0.01$ \\
S4 & 045020.69 & -025815.6 & $68 \pm 7$ & $35 \pm 2$ & $1.7 \pm 0.2$ & $0.7 \pm 0.1,3.4 \pm 0.1^{\dagger}$ \\
S5 & 045017.82 & -025717.6 & $11 \pm 1$ & $4.9 \pm 0.3$ & $0.6 \pm 0.1$ & $0.9 \pm 0.1,2.3 \pm 0.2^{\dagger}$ \\
A & 045023.36 & -030233.6 & - & $3.2 \pm 0.2$ & $1.5 \pm 0.2$ & $0.8 \pm 0.2$ \\
B & 045016.42 & -030154.6 & - & $0.96 \pm 0.10$ & $1.5 \pm 0.2$ & $-0.5 \pm 0.2$ \\
\hline
\end{tabular}

$\dagger$ The two spectral indices are between $235-610$ and $610-1500 \mathrm{MHz}$, respectively.

\subsection{GMRT}

PLCK G200.9-28.2 was observed with the GMRT using the dual frequency mode (610 and $235 \mathrm{MHz}$ ). This mode allows simultaneous recording of data at 610 and $235 \mathrm{MHz}$ with one polarization at each frequency. The data reduction was carried out using NRAO Astronomical Image Processing System (AIPS). The data at 610 and $235 \mathrm{MHz}$ were saved in separate FITS files and the standard steps of data reduction such as flagging (removal of bad data), calibration and imaging and self-calibration were followed. The standard cal- ibrators 3C48 and 3C147 were used for flux and bandpass calibration and the sources $0409-179$ and $0521+166$ were used for phase calibration. The calibrated target source data were examined and bad data were removed. The final visibilities on the target were then split from the multi-source file and averaged in frequency appropriately to avoid being affected by bandwidth smearing and at the same time to reduce the size of the data. The frequency averaged data were then imaged in AIPS. A few iterations of phase selfcalibration and one iteration of amplitude and phase self- 


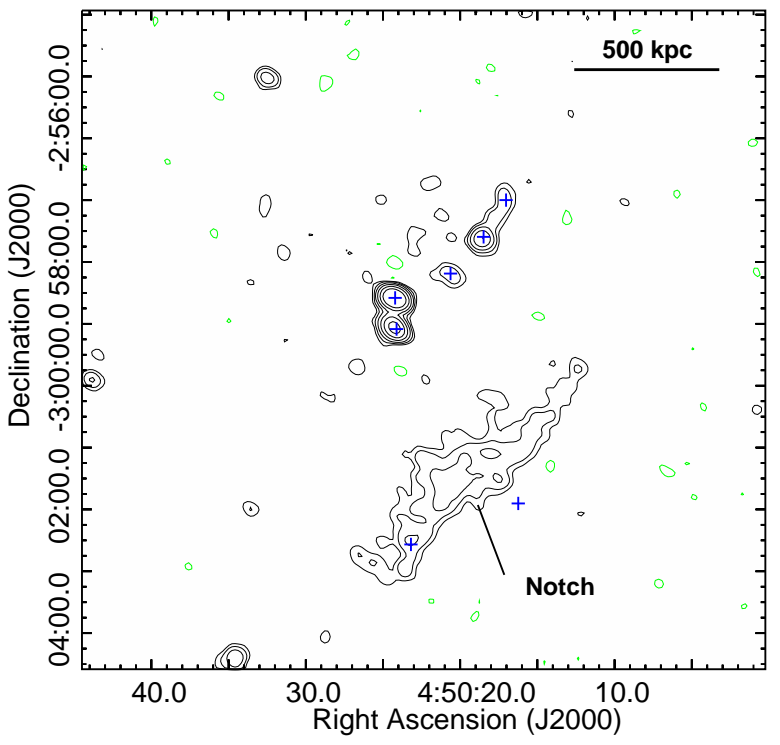

(a) GMRT $235 \mathrm{MHz}$

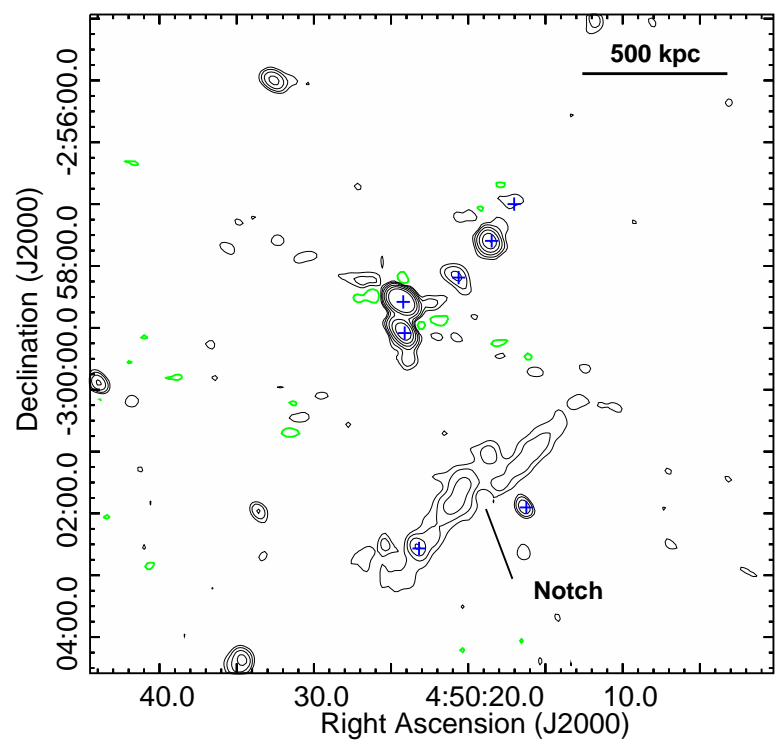

(b) VLA $1500 \mathrm{MHz}$

Figure 2. (a) - GMRT $235 \mathrm{MHz}$ image with $\sigma_{\mathrm{rms}}=0.39 \mathrm{mJy} \mathrm{beam}^{-1}$ is and resolution of $14.7^{\prime \prime} \times 12.8^{\prime \prime}$, position angle $57.6^{\circ}$ is shown in contours. (b) - VLA $1.5 \mathrm{GHz}$ image with a resolution of $14.5^{\prime \prime} \times 10.0^{\prime \prime}$, position angle $33.7^{\circ}$ and $\sigma_{\text {rms }}=0.06 \mathrm{mJy}$ beam ${ }^{-1}$ is shown in contours. In panels a and $\mathrm{b}$, the black are positive and the green are negative contour levels. The plus signs mark the discrete sources labelled in Fig. 1.

calibration were carried out. At an intermediate stage in self-calibration, when most of the sources were cleaned, the clean components were subtracted from the visibilities. The residuals were clipped to excise the low level RFI and the clean components were added back. These visibilities were used for further iterations of self-calibration. The final images were produced using robust " 0 " weights and the rms in the same has been reported in Table 2. Restriction of uv-range was used to produce images with lower resolutions for the analysis of extended emission. The images were corrected for the GMRT primary beam using the polynomial approximation to the beam using the task 'PBCOR'. A conservative estimate of amplitude calibration error $\left(\sigma_{\text {amp }}\right)$ of 10 per cent at both 235 and $610 \mathrm{MHz}$ was considered in calculating the errors on the measured flux densities of the sources. The AIPS task TVSTAT was used for measuring the total flux density of extended sources and the task JMFIT was used for point sources. The error on the flux density $\left(\Delta S_{v}\right)$ of a source of strength $S_{v}$ at a frequency $v$, that has an extent of $N_{b}$, number of beams, is calculated as $\Delta S_{v}=\left[\left(\sigma_{\mathrm{amp}} * S_{v}\right)^{2}+\left(\sigma_{\mathrm{rms}} * \sqrt{N_{b}}\right)^{2}\right]^{1 / 2}$, where $\sigma_{\mathrm{rms}}$ is the rms noise in the image.

\section{$3.2 \quad$ VLA}

The VLA in C-configuration was used to observe PLCK G200.9-28.2 in the frequency band $1-2 \mathrm{GHz}$. The $1 \mathrm{GHz}$ bandwidth contained 16 frequency sub-bands, each with 64 channels. The pre-processed data provided by NRAO were downloaded and processed further in Common Astronomy Software Applications (CASA). The preprocessing involved flagging and calibration. Manual flagging was carried out to remove the residual bad data. From the 16 subbands five $(1,2,3,8,9)$ were flagged. Two chan- nels at the edges of each of the sub-bands were removed and 10 channels were averaged to a single channel. The data were averaged to $10 \mathrm{~s}$ to reduce the data volume. The final visibilities with 6 channels per sub-band and 10s samples were used for imaging. The CASA task 'clean', with the multi-scale multi-frequency synthesis (MS-MFS) imaging, was used. Three iterations of phase self-calibration were carried out. The rms in the image produced using robust $=$ 0 (weighting $=$ 'Briggs') for the visibilities is reported in Table 2. The task 'widebandpbcor' was used to correct for the effect of the primary beam of the VLA antennas. An amplitude calibration error of 10 per cent has been considered in calculating the errors on the measured flux densities.

\section{RADIO RESULTS}

The GMRT $610 \mathrm{MHz}$ image is presented in Fig. 1 along with an overlay on the Digitized Sky Survey R-band image. The GMRT $235 \mathrm{MHz}$ and the VLA $1500 \mathrm{MHz}$ images are presented in Fig. 2. The region of PLCK G200.9-28.2 consists of a few discrete sources and a large elongated source. The radio properties of these are reported in the following subsections.

\subsection{Discrete radio sources}

The discrete radio sources in the central region of the PLCK G200.9-28.2 field are named S1 - S5 and those near the large elongated radio source as $\mathrm{A}$ and $\mathrm{B}$. The discrete sources S1-S5 are all resolved at $610 \mathrm{MHz}$. The sources S1, S2 and S3 have optical counterparts. Sources S4 and S5 are both diffuse and have no optical counterparts in the DSS image. The integrated spectral indices of S1, S2, and S3 are in the 


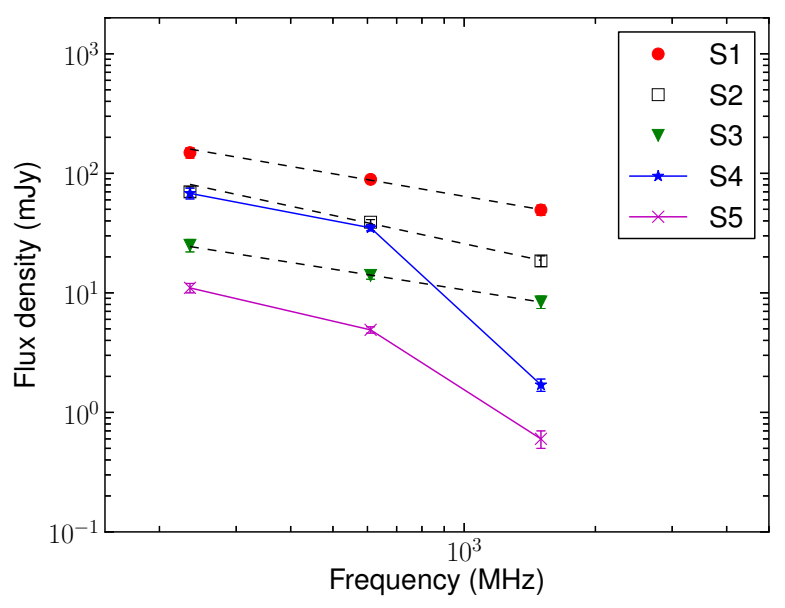

Figure 3. Integrated spectra of the sources $\mathrm{S} 1-\mathrm{S} 5$ in PLCKG200.9-28.2. The black dashed lines show the best fit spectra for S1, S2 and S3. The solid lines join the flux density points for S4 and S5 separately.

range 0.6 - 0.8 (Fig. 3, Table 3). The diffuse sources $\mathrm{S} 4$ and $\mathrm{S} 5$ show steepening in their spectra at higher frequencies. S4 and S5 may be radio lobes associated with S3. Towards the south of the cluster, embedded in the extended emission is the source A that can be identified with the galaxy 2MASX J04502353 - 0302367 (Fig. 1b). The unresolved source B lies beyond the edge of diffuse source towards the southwest, has no optical counterpart and has an inverted spectrum (Table 3).

\subsection{A radio relic}

There is an elongated, diffuse radio source located at a projected distance of about $0.9 \mathrm{Mpc}$ towards the south-west of the X-ray position of the cluster PLCK G200.9-28.2 (PC12, Fig. 1a). The discrete sources $\mathrm{A}$ and $\mathrm{B}$ in the region show no morphological connection like jets to the extended source at the highest resolution of $\sim 4^{\prime \prime}$ at $610 \mathrm{MHz}$ (in an image with uniform weights for the visibilities, not shown). We refer to this source as the radio relic. Assuming the relic to be at the redshift of the host cluster, the largest linear extent of the relic is $1020 \mathrm{kpc}$ and the largest width is $280 \mathrm{kpc}$. The properties of the relic are summarised in Table 4 .

\subsubsection{Integrated spectrum}

The flux density of the relic was measured in the each of the 235, 610, and $1500 \mathrm{MHz}$ image using identical regions. The flux density in the relic was measured in a region covering the extent of the relic which also included the source A. The flux density of A (Gaussian fit), which is the only point source in the region, was subtracted from it. The resulting flux densities of the relic and the spectral indices are reported in Table 4. The $150 \mathrm{MHz}$ image of the region of PLCK G200.9-28.2 available from the TIFR GMRT Sky Survey (TGSS ${ }^{1}$, PI S. Sirothia) was also used. The rms noise

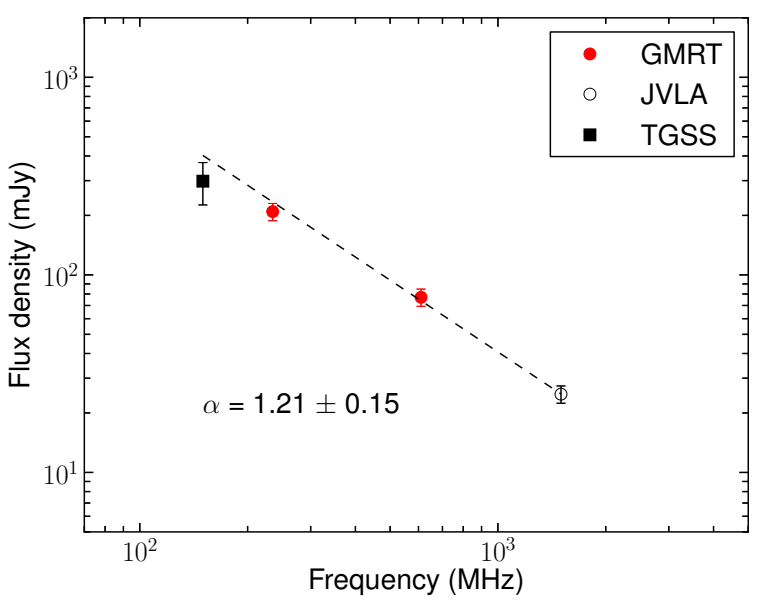

Figure 4. Integrated spectrum of the relic in PLCKG200.9-28.2. The dashed line shows the best fit to the spectrum.

in the image is $5 \mathrm{mJy}$ beam ${ }^{-1}$ (beam is $\sim 25^{\prime \prime}$ ). The flux density of the relic in this image, assuming a 20 per cent error, is reported (Table 4, Fig. 4). The integrated spectrum of the relic is a single power-law with a spectral index $1.21 \pm 0.15$.

\subsubsection{Spectral index map}

With radio observations at two or more frequencies with overlapping uv-coverages, spectral index maps of an extended source can be constructed. The steps to make the spectral index map between 235 and $610 \mathrm{MHz}$ are described briefly. The images at each frequency were made using uniform weights for the visibilities in the overlapping uv-range of $0.2-19 \mathrm{k} \lambda$. These were corrected for the effect of the respective primary beams and convolved to a common resolution of $16.4^{\prime \prime} \times 16.4^{\prime \prime}$. The rms noise $\left(\sigma_{\text {rms }}\right)$ in the resulting images were 0.16 and $0.48 \mathrm{mJy}_{\text {beam }}{ }^{-1}$ at 610 and $235 \mathrm{MHz}$ respectively. The regions of the image with flux densities below $3 \sigma_{\text {rms }}$ were blanked. The resulting images were used in the task 'COMB' to make spectral index maps. The spectral index and the corresponding noise map are presented in Fig. 5. The spectral indices at the south western edge (outer edge) of the relic are flatter $(\sim 0.7)$ and steepen towards the north east $(0.9-1.3)$.

\section{X-RAY ANALYSIS}

\subsection{Data analysis}

PLCK G200.9-28.2 was observed for $17 \mathrm{ks}$ with XMMNewton in February 2011 (ObsID 0658200801). The EPIC data were reduced with SAS version $11.0 .0^{2}$, primarily using the Extended Source Analysis Software (XMM-ESAS) subpackage. We follow the analysis method introduced by Snowden et al. (2008) for EPIC MOS. For EPIC pn, the methods are described in (e.g., Bulbul et al. 2012; Giacintucci et al. 2013). A brief summary of the analysis is presented here. A filtered event list is created by removing 


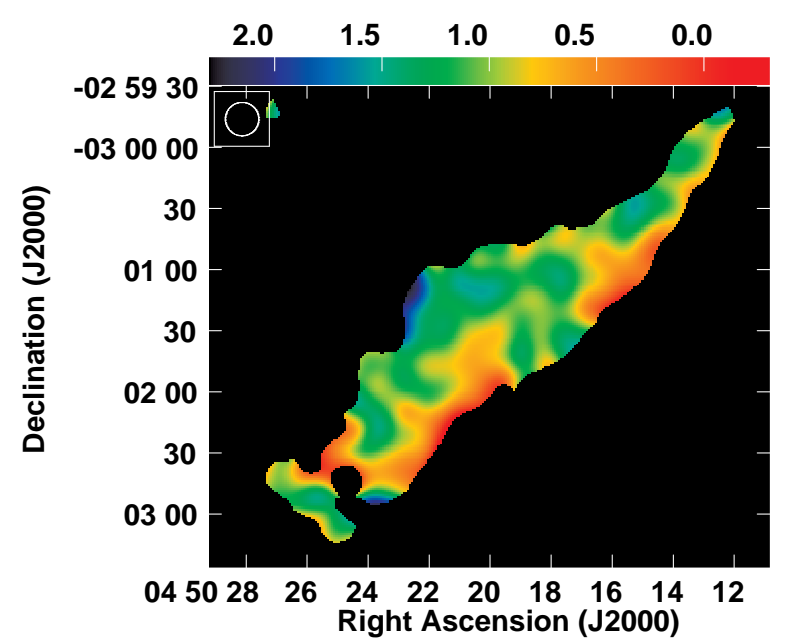

(a) Spectral index map

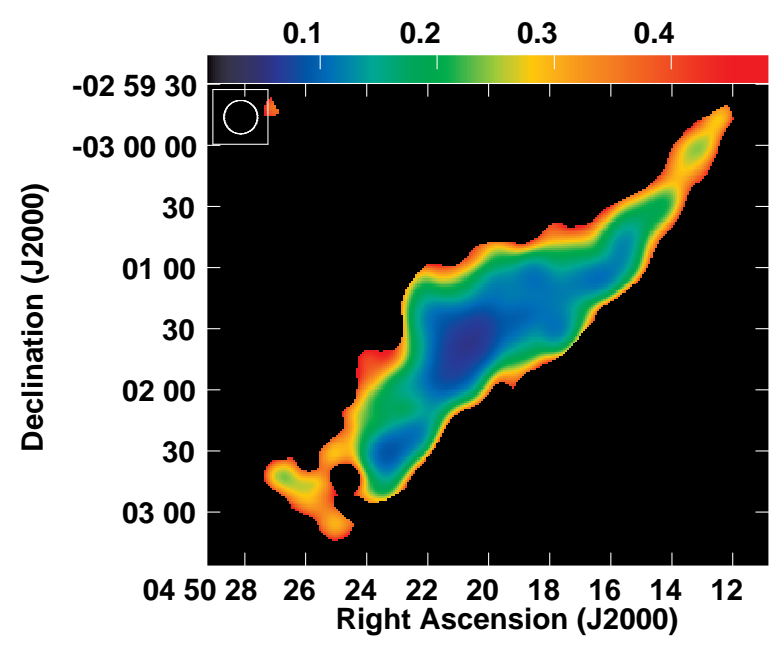

(b) Spectral index error map

Figure 5. (a) - The spectral index map of the radio relic between 235 and $610 \mathrm{MHz}$. (b) - The corresponding noise map. The resolution of the maps shown in upper left corner of each panel is $16.4^{\prime \prime} \times 16.4^{\prime \prime}$.

Table 4. Properties of the radio relic.

\begin{tabular}{ll}
\hline $\mathrm{RA}_{\mathrm{J} 2000}^{\ddagger}$ & $04 \mathrm{~h} 50 \mathrm{~m} 20.7 \mathrm{~s}$ \\
$\mathrm{DEC}_{\mathrm{J} 2000}$ & $-03^{\circ} 01^{\prime} 37.6^{\prime \prime}$ \\
$\mathrm{LLS}^{\dagger}$ & $1020 \mathrm{kpc}$ \\
Largest Width & $280 \mathrm{kpc}$ \\
$S_{150 \mathrm{MHz}}$ & $298 \pm 82 \mathrm{mJy}$ \\
$S_{235 \mathrm{MHz}}$ & $209 \pm 21 \mathrm{mJy}$ \\
$S_{610 \mathrm{MHz}}$ & $77 \pm 8 \mathrm{mJy}$ \\
$S_{1500 \mathrm{MHz}}$ & $24.9 \pm 2.5 \mathrm{mJy}$ \\
$\alpha$ & $1.21 \pm 0.15$ \\
$P_{1.4 \mathrm{GHz}}^{*}$ & $(3.9 \pm 0.4) \times 10^{24} \mathrm{~W} \mathrm{~Hz}^{-1}$ \\
Mach number, $M_{\text {int }}$ & $2.8 \pm 0.5$ \\
Mach number, $M_{\text {edge }}$ & $3.3 \pm 1.8$ \\
\hline
\end{tabular}

$¥ \mathrm{RA}_{\mathrm{J} 2000}$ and $\mathrm{DEC}_{\mathrm{J} 2000}$ are the coordinates of the peak at 610 $\mathrm{MHz} .{ }^{\dagger}$ Largest linear size. ${ }^{*}$ Power at $1.4 \mathrm{GHz}$ is calculated by extrapolating the $S_{1500 \mathrm{MHz}}$ to $1.4 \mathrm{GHz}$ using the spectral index,

periods of excessive count rate in the light curve caused by proton flaring events (retaining $16.1 \mathrm{ks}, 15.7 \mathrm{ks}$, and $4.8 \mathrm{ks}$ of the exposures for MOS1, MOS2, and pn, respectively), excluding non-functioning MOS CCDs (in this case CCDs 4, 5, and 6 for MOS1), and identifying and excluding point sources. Unfortunately, all data were highly contaminated by proton flaring, so we exclude periods with high incidence of flares and keep those with only lower level flaring.

Spectra and images are then extracted for source regions and the quiescent particle background, the latter of which are derived from a database of filter wheel closed observations that are matched to data using the unexposed corners of the chips. Remaining background components (residual soft proton contamination, instrumental lines, solar wind charge exchange (SWCX) emission, and the cosmic foreand backgrounds from the local hot bubble, the Galaxy, and extragalactic unresolved point sources) are all explicitly modeled and determined empirically during spectral fits in XSPEC. While robust best-fits of these components can be trickier to obtain in short exposures such as these, the small angular extent of the cluster leaves much of the FOV available for background modeling. We fix the absorbing column to the Galactic value $\left(n_{H}=3.9 \times 10^{20} \mathrm{~cm}^{-2}\right)$ and the redshift of the cluster $(z=0.22)$ to that reported previously in PC12. The local hot bubble (unabsorbed $k T=0.1 \mathrm{keV}$ ) and Galactic emission (absorbed, two temperature model with $k T \mathrm{~s}$ of $0.1 \mathrm{keV}$ and $0.3 \mathrm{keV}$ ) are further constrained by a ROSAT all sky survey spectrum from an annular region with radius $1 \mathrm{deg}^{2}$ centered on the cluster position, obtained with the online X-ray background tool ${ }^{3}$. Due to the high level of flaring, the soft proton component is modeled as a broken power law with indices $\Gamma$ and break energies $E_{\mathrm{b}}$ left free, leading to consistent values between all instruments with hard $(\Gamma \sim 0.3)$ indices below $E_{\mathrm{b}} \sim 1.5 \mathrm{keV}$ and softer $(\Gamma \sim 1.0)$ indices above $E_{\mathrm{b}}$. Also, no SWCX lines are evident in any of the spectra.

For image analysis, we combine the data, background, and properly scaled exposure maps (in equivalent MOS2 medium filter count rates) from all the three instruments. Separate background images include the contributions of the non-X-ray particle background (scaled and taken from stowed exposures), the soft proton background (scaled and taken from the ESAS CALDB), and the cosmic X-ray background, of both diffuse and unmasked point source emission, as modeled during spectral fitting (convolved with exposure maps). When extracting a profile, the size of a bin is increased until 50 net (data minus background) source counts are accumulated, which is then divided by the average exposure within the bin and area excluding masked pixels.

\subsection{X-ray Results}

The XMM-Newton image in $0.4-1.4 \mathrm{keV}$ band is presented in Fig. 6a. Due to the residual soft proton contamination, we restrict our spatial analysis to this softer band to maximize signal-to-noise; however, note that all spectra are fit

\footnotetext{
${ }^{3}$ http://heasarc.gsfc.nasa.gov/cgi-bin/Tools/xraybg/xraybg.pl
} 
over the full $0.3-12 \mathrm{keV}$ band so that temperatures are well constrained. The morphology in the image can be divided into northern and southern sub-clusters. The relic is at the south-western periphery skirting the southern sub-cluster. We find temperatures of $5.3_{-0.9}^{+1.0} \mathrm{keV}$ and $6.1_{-1.2}^{+1.9} \mathrm{keV}$ for the northern and southern sub-clusters, respectively. While the southern subcluster temperature may be biased high by a shock related to the radio relic, and/or the northern subcluster temperature may be biased low due to a cool core, additional X-ray observations are required to say more. We measure the total average temperature of the entire cluster to be $5.6 \pm 0.8 \mathrm{keV}$, with an unabsorbed luminosity of $L_{X[0.1-2.4 \mathrm{keV}]}=(1.05 \pm 0.05) \times 10^{44} \mathrm{erg} \mathrm{s}^{-1}$. This is somewhat hotter than the value found by PC12 of $4.5 \pm 0.7 \mathrm{keV}$, likely a consequence of the high background and difficulty in modeling it accurately. This mild discrepancy should be taken as a demonstration of the impact of systematic uncertainties. Our slightly higher value of $L_{X}$ is likely due to including the southernmost emission from the cluster, which falls outside the $R_{500}$ radius considered by PC12. The abundance is constrained to be no higher than 0.38 relative to solar, consistent with clusters generally.

In Fig. 6a, we extract a surface brightness profile within a circular wedge that roughly aligns with the total curvature of the relic. The Fig. $6 \mathrm{~b}$ shows the surface brightness profile along with the total background level (solid gray boxes, indicating $1 \sigma$ uncertainties) and the location of the radio relic (hatched region). The edge of the relic falls well below the background level, making any estimate of a surface brightness jump impossible, although there is a large drop in surface brightness just outside the relic. Since the preshock region is deep in the background, we cannot formally constrain the density jump, and hence estimate Mach number from these data. We model a density jump in the surface brightness profile assuming a power law density profile $\left(\propto n_{e}^{\Gamma}\right)$ with a discontinuity fixed at the location of the edge of the relic. If we fix the index of the density profile to $\Gamma=1.7$, the best-fit value on both sides of the potential shock, the density jump is constrained to be less than a factor of 2 (90 per cent confidence), or $M<1.8$. Whether the X-ray estimate of the Mach number is actually in tension with the radio estimate, however, will be confirmed by deeper observations. Assuming no jump at the radio relic location, the profile can be described by a classic $\beta$-model with core radius of $1.0_{-0.4}^{+0.9}$ arcmin, $\beta=0.6_{-0.1}^{+0.4}$, and central surface brightness of $\left(3.9_{-0.7}^{+1.0}\right) \times 10^{-3}$ counts s$^{-1} \operatorname{arcmin}^{-2}$.

\section{DISCUSSION}

\subsection{Relic power, size and cluster mass}

The correlation between the relic power at $1.4 \mathrm{GHz}\left(P_{1.4 \mathrm{GHz}}\right)$ and the host cluster mass has been reported for the sample of known single and double radio relics (de Gasperin et al. 2014). The PLCK G200.9-28.2 relic plotted in the $P_{1.4 \mathrm{GHz}}-$ mass plane along with other known radio relics is shown in Fig. 7. The literature data presented in de Gasperin et al. (2014) have been revised for relics with unknown spectral indices ${ }^{4}$ and updated to include the radio relics in the clusters A3527-bis and PSZ1 G108.18-11.53 (de Gasperin et al. $2015,2017)$. The revised scaling relation has a slope of $2.76 \pm 0.37$ and an intercept of $-16.2 \pm 5.3$ (Fig. 7).

PLCK G200.9-28.2 has the lowest mass among the clusters with single relics and is the third lowest mass cluster among all the radio relic clusters. The two clusters with masses less than PLCK G200.9-28.2 are Abell 3365 (van Weeren et al. 2011) and Abell 3376 (Bagchi et al. 2006; Kale et al. 2012), both with double relics. As compared to the predictions of the simulations by Nuza et al. (2012), the probability of finding a radio relic in a cluster of mass $\sim 3.3 \times 10^{14} \mathrm{M}_{\odot}$ and redshift $\sim 0.2$ is $<$ few per cent with the current instruments (de Gasperin et al. 2014). The case of the PLCK G200.9-28.2 relic provides an example of an even lower mass cluster with a powerful radio relic. The sample of single relics does not follow the radio power-mass scaling and PLCK G200.9-28.2 is a factor of 10 more powerful in radio than expected from the correlation.

We investigated whether the largest linear sizes of the single relics show scaling with radio power, mass or redshift. In Fig. 7, the sizes of the circles denoting single relics are scaled according to their largest linear sizes. We point out that among the powerful $\left(>10^{24} \mathrm{~W} \mathrm{~Hz}^{-1}\right)$ single radio relics, PLCK G200.9-28.2 has the smallest size. However, there is no trend in size with respect to the host cluster mass, redshift and relic radio power. The effects of projection may be the important factors in the apparent sizes of the relics.

\subsection{A merger shock ?}

The cluster PLCK G200.9-28.2 in X-rays shows a disturbed morphology indicating an ongoing merger. There is a northern clump of gas that extends with a lower brightness emission to the south reaching the location of the relic (Fig. 6). The spectral index map of the relic shows flatter spectrum emission towards the outer edge and gradual steepening towards the inner side strongly indicating an underlying merger shock. Under the assumptions of DSA, if $\alpha_{\text {inj }}$ is the spectral index at injection then the Mach number of the shock is given by (e.g. Blandford \& Eichler 1987),

$$
M=\sqrt{\frac{2 \alpha_{\mathrm{inj}}+3}{2 \alpha_{\mathrm{inj}}-1}} .
$$

In the case of PLCK G200.9-28.2 relic, the $\alpha_{\text {inj }}$ is the spectral index at the outer edge of the relic, equal to $0.7 \pm 0.2$ (Fig. 5). This implies, $M=3.3 \pm 1.8$ for the relic. In a scenario where there is continuous injection at the shock, a spectral index of $\alpha_{\mathrm{inj}}+0.5$ is expected. The integrated spectral index of $1.21 \pm 0.15$ is consistent with this expectation and implies $M=$ $3.2 \pm 1.3$. This relic is one of the cases where the integrated spectra and the injection spectral index are consistent with continuous injection scenario.

If a shock is detected in X-rays, then a Mach number independent of the detection in radio can be estimated. The X-ray derived Mach numbers have been found

4 The spectral index of -1.0 was mistakenly used in de Gasperin et al. (2014) to calculate the radio powers of the relics with unknown spectral indices. We have used the correct value of 1.0 and revised the radio powers. 


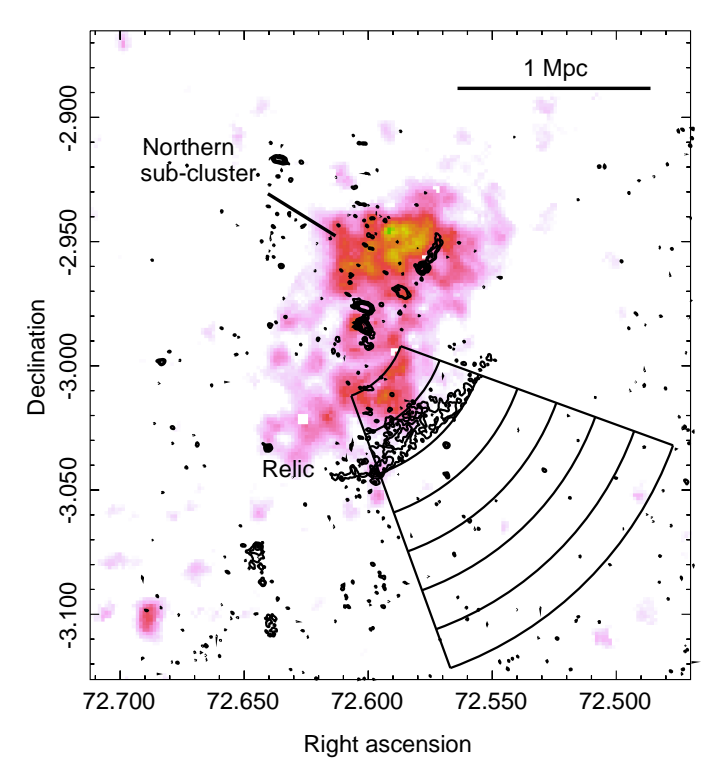

(a) XMM-Newton $0.4-1.4 \mathrm{keV}$ with $610 \mathrm{MHz}$ contours.

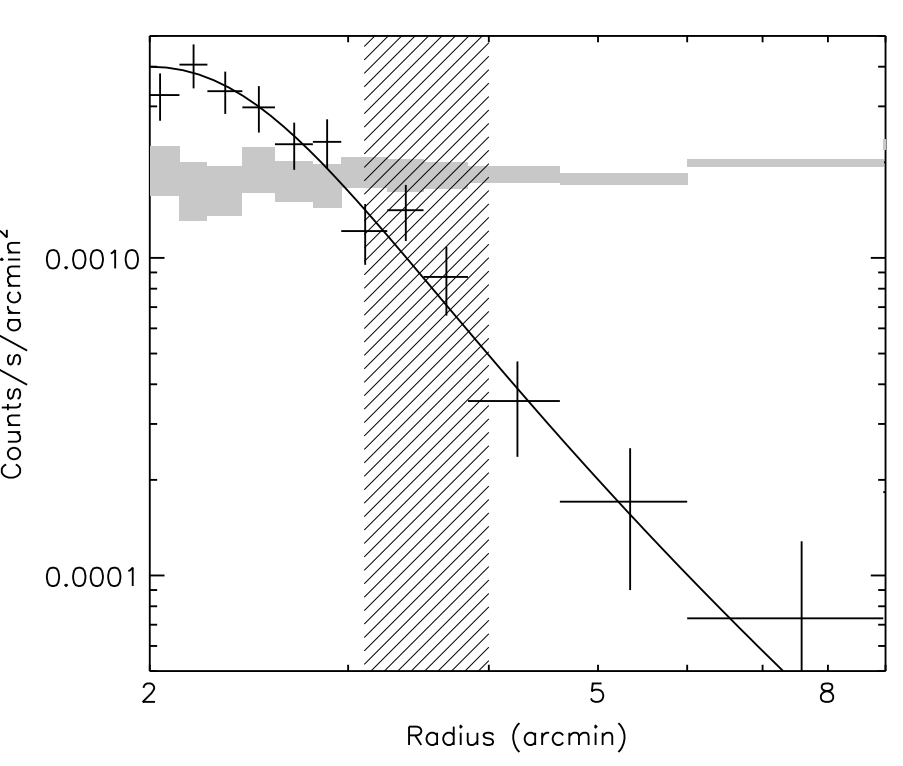

(b) Surface brightness profile.

Figure 6. (a)- XMM-Newton 0.4-1.4 keV adaptively smoothed, greyscale image with overlays of the radio relic (black contours same as Fig. 1b) and of the circular sector used to extract the X-Ray surface brightness profile in the same energy band. Each arc corresponds to a width of $1^{\prime}$. (b)- 0.4-1.4 keV surface brightness profile (crosses, 1-sigma errors shown). The hatched region indicates the location of the relic and the gray bars show the estimated background level in each bin. The solid line shows a beta profile with no density jump. Because the XMM-Newton data are not sufficiently sensitive to detect the emission beyond the relic, a density jump cannot be ruled out.

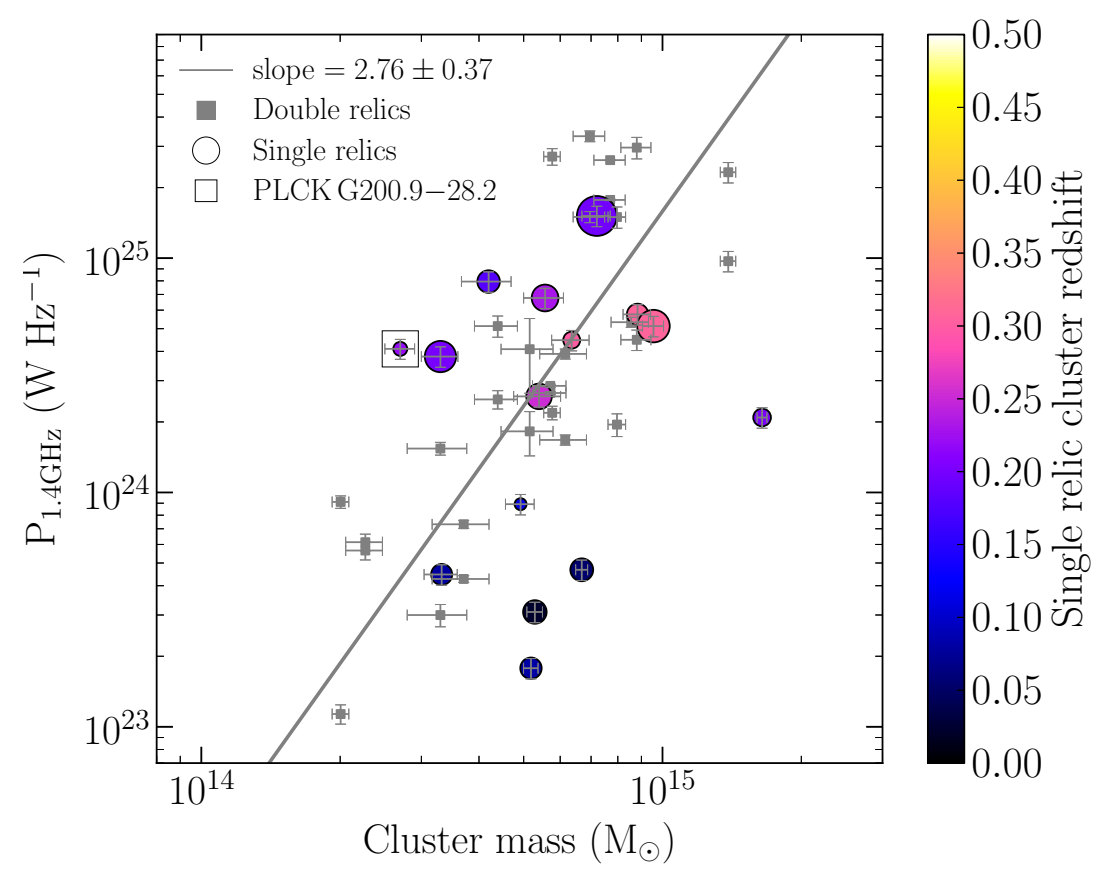

Figure 7. The $1.4 \mathrm{GHz}$ power of radio relics is plotted against the host cluster mass. The circles show the single relics, including PLCK G200.9-28.2. The sizes of the circles are scaled according to the largest linear sizes of the relics and the colour denotes the redshift. PLCK G200.9-28.2 relic is highlighted with an empty square. The grey squares show the double relics. The best fitting scaling relation for the double relics is shown by the dashed line. The data for the known relics from de Gasperin et al. (2014, 2015, 2017) and the best fitting slope have been revised and plotted (Sec. 6.1). PLCK G200.9-28.2 has the lowest mass among the hosts of single relics. The PLCK G200.9-28.2 relic has the smallest size among the single relics with radio powers greater than $10^{24} \mathrm{~W} \mathrm{~Hz}^{-1}$. 
consistent with those from radio spectra in some relics (Akamatsu \& Kawahara 2013) but may also have a mismatch (Ogrean et al. 2013). From the XMM-Newton X-ray data on PLCK G200.9-28.2, a Mach number of $<1.8$ is expected presuming a density jump, which is less than that from the radio spectral indices. Our recently approved Chan$d r a$ observations will provide precise measurements of the Mach number.

The morphology of PLCK G200.9-28.2 relic is overall arc-like with a notch at the outer edge (Fig. 1). The notch is a low brightness region which breaks the smoothness of the outer edge of the relic. It is prominent in higher frequency images as compared to that at $235 \mathrm{MHz}$. Such features can indicate the changes in the underlying physical conditions in the shock. It can also be an effect of a density change due to an infalling structure such as proposed in some simulations (e.g. Paul et al. 2011).

\subsection{X-ray and SZ position offset}

Cluster mergers can stir the ICM and lead to complex distributions of density and temperatures. The X-ray surface brightness traces regions of high electron densities $\left(\propto n_{e}^{2}\right)$ and the SZ is sensitive to the pressure $\left(\propto n_{e} T\right)$ along the line of sight. An offset in the peaks of these signals can be used as an indicator of the density and temperature distribution in the disturbed ICM. There are examples of merging clusters that show presence of X-ray -SZ offsets, such as Abell 2146 (AMI Consortium et al. 2011) and Bullet cluster (Malu et al. 2010). Simulations have shown that the offset is sensitive to initial relative velocity of the merging clusters and the mass ratio (e. g. Molnar et al. 2012; Zhang et al. 2014). The cluster PLCK G200.9-28.2 discussed in this work has its X-ray peak and the Planck detection peak offset by 3.4', which is the extreme in the Planck sample (PC12). The Planck SZ positions have a mean and median errors of 1.5 and 1.3' respectively (Planck Collaboration et al. 2013). The $\mathrm{X}$-ray peak is located at the northern sub-cluster (Fig. 1a and 6a) and the Planck SZ position is separated from it by $3.4^{\prime}(700 \mathrm{kpc})$ in the direction of the radio relic. Due to the presence of a shock at the relic, the region is expected to be overpressured and thus may result in shifting the peak of the SZ-signal. The offset in the direction of the relic indicates possible physical origin for the offset in addition to the position reconstruction uncertainty of Planck. Based on the results of simulations, the offset can be explained as a result of two comparable mass sub-clusters with mass ratio between 1 and 3 (Zhang et al. 2014). Deep optical observations tracing the galaxy distribution in this cluster will be useful to measure the mass ratios of the sub-cluster masses. Due to the large uncertainty in the Planck position we cannot analyse the offsets for a statistical sample of merging clusters. However, the offset in the X-ray and SZ positions opens an additional probe for understanding the properties of merging galaxy clusters.

\section{CONCLUSIONS}

We have presented the discovery of a single arc-like relic in the galaxy cluster PLCK G200.9-28.2 discovered by the Planck satellite. This cluster has the lowest mass among the clusters known to host single arc-like radio relics. The main results and conclusions from our work are as follows:

(i) The GMRT 235 and $610 \mathrm{MHz}$ and VLA $1500 \mathrm{MHz}$ images confirm the presence of a radio relic of size $\sim 1 \times 0.28$ $\mathrm{Mpc}$ located at a projected distance of $0.9 \mathrm{Mpc}$ from the centre of the cluster PLCKG200.9-28.2.

(ii) The XMM-Newton X-ray images show merging northern and southern sub-clusters in this cluster and the relic is located at the south-western periphery of the cluster.

(iii) The radio relic has an integrated spectral index of $1.21 \pm 0.15$. The $235-610 \mathrm{MHz}$ spectral index map shows steepening from outer to inner edge of the relic. This is consistent with expectations for a relic generated by an underlying merger shock.

(iv) The radio relic has a $1.4 \mathrm{GHz}$ power of $(3.9 \pm 0.4) \times 10^{24}$ $\mathrm{W} \mathrm{Hz}{ }^{-1}$. The PLCK G200.9-28.2 is the lowest mass single relic cluster known. The radio relic is the smallest in size in the sample of single relics with radio power $>10^{24} \mathrm{~W} \mathrm{~Hz}^{-1}$.

(v) The analysis of the X-ray brightness profile along relic shows the possibility of a putative shock. Future deep $C$ handra observations will be able to characterize the shock in X-rays.

(vi) The cluster PLCK G200.9-28.2 has the extreme offset of $3.4^{\prime}(700 \mathrm{kpc})$ in the positions of the peak in X-ray and in Planck SZ. The Planck position is offset toward the position of the relic where high pressure region may be expected due to merger shock. The X-ray - SZ position offset may be of physical origin and an important probe of merging cluster with future better measurements in SZ positions.

\section{ACKNOWLEDGEMENTS}

We thank the anonymous referee for comments that helped to improve the paper. RK acknowledges the support from DST-INSPIRE Faculty Award from the Department of Science and Technology, Government of India. GB, TV, RC acknowledge partial support from PRIN-INAF 2014. F.d.G. is supported by the VENI research programme with project number 1808, which is financed by the Netherlands Organisation for Scientific Research (NWO). We thank the staff of the GMRT that made these observations possible. GMRT is run by the National Centre for Radio Astrophysics of the Tata Institute of Fundamental Research. The National Radio Astronomy Observatory is a facility of the National Science Foundation operated under cooperative agreement by Associated Universities, Inc. Based on observations obtained with XMM-Newton, an ESA science mission with instruments and contributions directly funded by ESA Member States and NASA. Basic research in radio astronomy at the Naval Research Laboratory is supported by 6.1 base funding. This research has made use of the NASA/IPAC Extragalactic Database (NED) which is operated by the Jet Propulsion Laboratory, California Institute of Technology, under contract with the National Aeronautics and Space Administration.

\section{REFERENCES}

AMI Consortium et al., 2011, MNRAS, 414, 3751

Akamatsu H., Kawahara H., 2013, PASJ, 65, 16 
Bagchi J., Durret F., Neto G. B. L., Paul S., 2006, Science, 314, 791

Bagchi J., et al., 2011, ApJ, 736, L8

Basu K., Vazza F., Erler J., Sommer M., 2016, A\&A, 591, A142

Blandford R., Eichler D., 1987, Phys. Rep., 154, 1

Bonafede A., et al., 2012, MNRAS, 426, 40

Brunetti G., Jones T. W., 2014, International Journal of Modern Physics D, 23, 30007

Bulbul G. E., Smith R. K., Foster A., Cottam J., Loewenstein M., Mushotzky R., Shafer R., 2012, ApJ, 747, 32

Cohen A. S., Lane W. M., Cotton W. D., Kassim N. E., Lazio T. J. W., Perley R. A., Condon J. J., Erickson W. C., 2007, AJ, 134, 1245

Condon J. J., Cotton W. D., Greisen E. W., Yin Q. F., Perley R. A., Taylor G. B., Broderick J. J., 1998, AJ, 115, 1693

Enßlin T. A., Biermann P. L., Klein U., Kohle S., 1998, A\&A, 332,395

Farnsworth D., Rudnick L., Brown S., Brunetti G., 2013, ApJ, 779,189

Giacintucci S., et al., 2008, A\&A, 486, 347

Giacintucci S., Kale R., Wik D. R., Venturi T., Markevitch M., 2013, ApJ, 766, 18

Giovannini G., Feretti L., 2000, New A, 5, 335

Guo X., Sironi L., Narayan R., 2014, ApJ, 797, 47

Hoeft M., Brüggen M., 2007, MNRAS, 375, 77

Kale R., Dwarakanath K. S., Bagchi J., Paul S., 2012, MNRAS, 426,1204

Kale R., Venturi T., Giacintucci S., Dallacasa D., Cassano R., Brunetti G., Macario G., Athreya R., 2013, A\&A, 557, A99

Kale R., et al., 2015, A\&A, 579, A92

Kang H., Jones T. W., 2005, ApJ, 620, 44

Kang H., Ryu D., 2015, ApJ, 809, 186

Kang H., Ryu D., Jones T. W., 2012, ApJ, 756, 97

Kempner J. C., Sarazin C. L., 2001, ApJ, 548, 639

Malu S. S., Subrahmanyan R., Wieringa M., Narasimha D., 2010, ArXiv e-prints 1005.1394,

Markevitch M., 2010, ArXiv e-prints 1010.3660,

Markevitch M., Govoni F., Brunetti G., Jerius D., 2005, ApJ, 627,733

Matsukiyo S., Ohira Y., Yamazaki R., Umeda T., 2011, ApJ, 742,47

Molnar S. M., Hearn N. C., Stadel J. G., 2012, ApJ, 748, 45

Nuza S. E., Hoeft M., van Weeren R. J., Gottlöber S., Yepes G., 2012, MNRAS, 420, 2006

Ogrean G. A., Brüggen M., van Weeren R. J., Röttgering H., Croston J. H., Hoeft M., 2013, MNRAS, 433, 812

Ogrean G. A., Brüggen M., van Weeren R. J., Burgmeier A., Simionescu A., 2014, MNRAS, 443, 2463

Orrú E., Murgia M., Feretti L., Govoni F., Brunetti G., Giovannini G., Girardi M., Setti G., 2007, A\&A, 467, 943

Paul S., Iapichino L., Miniati F., Bagchi J., Mannheim K., 2011, ApJ, 726, 17

Planck Collaboration et al., 2011a, A\&A, 536, A8

Planck Collaboration et al., 2011b, A\&A, 536, A9

Planck Collaboration et al., 2012, A\&A, 543, A102

Planck Collaboration et al., 2013, A\&A, 550, A130

Planck Collaboration et al., 2015, A\&A, 581, A14

Rengelink R. B., Tang Y., de Bruyn A. G., Miley G. K., Bremer M. N., Roettgering H. J. A., Bremer M. A. R., 1997, A\&AS, 124

Snowden S. L., Mushotzky R. F., Kuntz K. D., Davis D. S., 2008, A\&A, 478, 615

Stroe A., van Weeren R. J., Intema H. T., Röttgering H. J. A., Brüggen M., Hoeft M., 2013, A\&A, 555, A110

Stroe A., et al., 2016, MNRAS, 455, 2402

Trasatti M., Akamatsu H., Lovisari L., Klein U., Bonafede A., Brüggen M., Dallacasa D., Clarke T., 2015, A\&A, 575, A45

Zhang C., Yu Q., Lu Y., 2014, ApJ, 796, 138 de Gasperin F., van Weeren R. J., Brüggen M., Vazza F., Bonafede A., Intema H. T., 2014, MNRAS, 444, 3130

de Gasperin F., Intema H. T., van Weeren R. J., Dawson W. A., Golovich N., Wittman D., Bonafede A., Brüggen M., 2015, MNRAS, 453, 3483

de Gasperin F., et al., 2017, A\&A, 597, A15

van Weeren R. J., et al., 2009, A\&A, 506, 1083

van Weeren R. J., Röttgering H. J. A., Brüggen M., Hoeft M., 2010, Science, 330, 347

van Weeren R. J., Brüggen M., Röttgering H. J. A., Hoeft M., Nuza S. E., Intema H. T., 2011, A\&A, 533, A35

van Weeren R. J., Röttgering H. J. A., Intema H. T., Rudnick L., Brüggen M., Hoeft M., Oonk J. B. R., 2012, A\&A, 546, A124

This paper has been typeset from a $\mathrm{T}_{\mathrm{E}} \mathrm{X} / \mathrm{LAT}_{\mathrm{E}} \mathrm{X}$ file prepared by the author. 